\title{
Regression Analysis via Dynamic Programming: II. Computational Results
}

\author{
Robert Kalaba \\ Harriet Natsuyama \\ Sueo Ueno \\ University of Southern California and Kyoto Computer Gakuin \\ hnatsu@earthlink.net
}

\section{Introduction}

In [1], we presented a new dynamic programming method for the solution of least squares regression problems. Here, we present the results of computational experiments verifying the robustness of the algorithm.

\section{Statement of the least squares problem}

The problem is, given the matrix $A$ and the vector $b$, we wish to determine the vector $x$ such that $|A x-b|^{2}$ is a minimum and the length of $x$ is as small as possible.

\section{Dynamic programming equations and algorithm}

We refer the reader to Part I. of this paper, this conference for the basic dynamic programming (DP) equations. The equations are used to develop an effective computational procedure (see below) for the $\alpha Q \beta R$ dynamic programming algorithm. The equation numbers refer to those in Part I. Please note the initialization and the updating (recurrence) of this stable initial value method. The procedure is easy to program in Fortran or other language and it runs quite efficiently while requiring only a modest amount of memory for current matrices and vectors and for the storage of certain vectors needed in the final sweep.

\section{Numerical results}

The dynamic programming method is successfully tested and utilized.

a. Test cases. The matrix is

$$
A=\left[\begin{array}{lll}
a_{1} & a_{2} & a_{3}
\end{array}\right]
$$

where

$a_{1}$ is the column vector $[1 ; 1 ; 1]$,

$a_{2}$ is $[2 ; 2 ; 2]$,

$a_{3}$ is $[0 ; 0 ; 1]$,

so that the second column is a multiple of the first, while the third is independent of the others. The correct solution is obtained in the following cases:

(a) The vector $b$ is $[1 ;-1 ; 0]$. The solution is $x=[0 ; 0 ; 0]$.

(b) The vector $b$ is $[0 ; 1 ; 1]$ has the solution $x=[0.1 ; 0.2 ; 0.5]$.

(c) The vector $b$ is $[1 ; 1 ; 0]$ and the solution is $x=[0.2 ; 0.4 ;-1.0]$. 


\section{Procedure for $\alpha Q \beta R$ Dynamic Programming Algorithm}

1. Input the $A$ matrix and the $b$ vector, set tolerance for $\alpha$

2. Sweep forward from columns 1 through $n$ and store all of the $\alpha$ and $\beta$ vectors

a. Column 1

i. Initialize $\alpha, Q_{1}$, and $R_{1}$ using Eqs. (9) - (11)

ii. Define $Q_{k-1}$ and $R_{k-1}$

b. Column $\mathrm{k}=2,3, \ldots, \mathrm{n}$

i. $\quad$ Compute $\alpha_{k}$ using Eq. (7) and store $\alpha_{k}$

ii. Test length of $\alpha$ against a tolerance

(a) If length is less than tolerance, use Eqs. (12), (13), (14) to compute $Q_{k}, \beta_{k}$, and $R_{k}$

and store $\beta_{k}$

(b) If length is greater than tolerance, use Eqs. (15), (16), (17) to compute $Q_{k}$ and $R_{k}$, and store; no $\beta_{k}$ is needed

iii. Shift current $Q_{k}$ and $R_{k}$ into $Q_{k-1}$ and $R_{k-1}$

3. Sweep backward and determine the components of the vector $x$ from the nth component to the first

a. Initialize the $b_{k}$ vector for $k=n$

i. $\quad$ Set $b_{n}=b$

ii. Compute $x_{n}$ using (20) or (21)

b. Component $k=n, n-1, n-2, \ldots, 1$

i. If $k=n$, skip this step; otherwise modify $b_{k}$ using Eq. (22)

ii. Test length of $\alpha$ against tolerance

(a) If length is less than tolerance, use Eq. (23a) to compute $x_{k}$

(b) If length is greater than tolerance, use Eq. (23b) to compute $x_{k}$

4. Output $x$ and other results as desired. 
b. Random matrix. In the first series of experiments, we take, for the matrix $A$, a random matrix produced by generating random numbers uniformly distributed on the $(-1,+1)$ interval. Matrix $A$ has four rows and three columns; vector $b$ has four components. We produce the computational solution vector $x$; this is our baseline case. Next, we study the performance of the algorithm when the column vectors of $A$ become more and more dependent.

Introducing a dependency coefficient, we produce other related matrices in which we take the third column to be the sum of (1-d) times the original third column and the sum of $d$ times the sum of the first two columns. By taking various values of the parameter $d$ between 0.00 (independent) to 0.9999 , we study the solution of regression problems in which the columns of the matrix grow increasingly dependent.

The dynamic programming algorithm compares favorably against the solution vectors produced by a commercial software package. A summary of the cases is presented in Table 1 . We found that the length of the vector grows and the determinant diminishes as the third column becomes decreasingly independent, i.e., more dependent.

We compare the dynamic programming results with those obtained using the built-in functions of Matlab. Both methods produce the same results, generally. However, differences are found when the dependency is large ( 0.99 or greater): dynamic programming obtains a finite length solution while Matlab finds an extremely long vector solution. [Matlab is a software package developed by The Math Works, Inc. and is licensed by Prentice Hall, Inc.]

c. Tolerance. In another series of experiments, we wish to study the effect of a tolerance use in a crucial branching test. The test occurs when determining whether a new column is independent of the previous ones or not. We find that the selection of the tolerance value is a delicate balancing act. Fortunately, a simple guideline has been found.

We study a matrix whose generalized inverse is analytically known. We use DP to compute the elements of the inverse as follows. We set the right-hand column vector $b$ equal to $[1 ; 0 ; 0]$ to find the first column of $A+$; then set $b=[0 ; 1$; $0]$ to obtain the second; finally set $b=[0 ; 0 ; 1]$ to determine the third. Adjoining the columns leads to the $2 \times 3$ matrix $A+$. The $A+$ determined this way is then compared to that obtained from a numerical evaluation of the formula.

Observe that the DP algorithm in no way requires that the inverse first be computed. We examine the computed inverses to learn the accuracy of the DP algorithm. We are especially concerned that, due to round-off errors and computer word length limitations, the testing small values for branching purposes may lead to the wrong branch, with consequent wrong solutions. We introduce a tolerance, tol, which we shall vary in this test. 
Table 1

Solution vector, its length and the determinant of the matrix as the dependency coefficient is increased.

(Matlab results are in italics.)

Depen.coeff. Solution $x \quad$ Length of $x$ Determinant

$\begin{array}{llll}0.00 & 0.8694 ;-.5494 ;-.0806 & 1.0642 & 0.8033 \\ 0.01 & -.8686 ;-.5486 ;-.0815 & 1.0620 & 0.7873 \\ 0.10 & -.8604 ;-.5405 ;-.0896 & 1.0405 & 0.6507 \\ 0.50 & & & \\ 0.99 & -.7887 ;-.4688 ;-.1613 & 0.8679 & 0.2008 \\ & -.4078 ;-.1024 ;-.5081 & 0.4350 & \\ 0.9999 & 7.114 ;-.4348 ;-8.0649 & 170.9386 & 8.032910^{\wedge}-5 \\ & -.4061 ;-.1016 ;-.5076 & 0.4329 & \\ & 805.5 ; 805.8 ;-806.5 & 1.948710^{\wedge} 6 & 8.032910^{\wedge}-9\end{array}$

The matrix we elect to study is

$$
A=\left[\begin{array}{ll}
1 & 1 \\
1 & 1+e \\
1 & 1-e
\end{array}\right],
$$

whose columns are nearly identical, the more so as the perturbation $e$ tends toward zero.

(a) When $e$ is exactly zero, i.e., when the columns are indeed non-independent, the generalized inverse is known to be

$$
A+=(1 / 6)\left[\begin{array}{lll}
1 & 1 & 1 \\
1 & 1 & 1
\end{array}\right] .
$$

In this case, the algorithm should take Branch 1.

(b) When e is not zero, the inverse is instead expressible as

$$
A+=\left[\begin{array}{ccc}
1 / 3 & 1 / 3-1 / 2 e & 1 / 3+1 / 2 e \\
0 & 1 / 2 e & -1 / 2 e \quad] .
\end{array}\right.
$$

Then $\alpha$ is not the null vector, and the current decision in the algorithm is to take Branch 2.

The dynamic programming algorithm branches when the $\alpha$ vector is "zero".

Computationally, we determine the square of the length of $\alpha$ and compare it with a tolerance tol. We are interested to know the pairings of $e$ and tol for which the incorrect branch selected. We vary $e$ and tol and study the branching when $\mathrm{k}$ $=2$, noting whether the right or wrong branch is selected. We also examine the elements computed for the inverse

We also compute the solution vector $x$ when the right-hand column vector is

$$
b=[0 ; 1 ; 1] .
$$

The correct solution is the column vector

$$
x=[1 / 6 ; 0] \text {. }
$$


The computational results for various non-zero values of $e$ and small values of tol are summarized in Table 2. In the table, when the "Inverse computed" is said to be "Incorrect", this indicates that the inverse obtained was that which would have been true if $e$ were exactly zero, which is not the case.

We find that, to obtain correct results, the tolerance must be set smaller than or equal to the square of $e$. When the branching is done correctly, then the solution vector is also computed correctly, even though the dynamic programming algorithm does not first compute the generalized inverse (unless it is desired). Of course, more experience is needed to determine how to set the tolerance when the interdependency of the columns of $A$ is not known.

The stability that has been predicted [2] has been observed, in that the calculations were carried out without exponential growth of errrors.

\section{Discussion}

Clearly, many more experiments and much more experience is desired for understanding the characteristics of the new algorithm. However, the results we've seen to date are extremely encouraging, and we have already begun to use the algorithm in applications such as estimating parameters in time-dependent transport problems [3].

\section{References}

[1] R. Kalaba, H. Natsuyama, and S. Ueno, "Regression analysis via dynamic programming: I. Theory," this conference.

[2] R.E. Bellman and R. E. Kalaba, Dunamic Programming and Modern Control Theory, Academic Press, New York, 1965.
[3] R. Kalaba, H. Natsuyama, and S. Ueno, "The estimation of parameters in time-dependent transport problems: Dynamic programming and associative memories," to appear in Computers and Mathematics with Applications 
Table 2.

Results of DP algorithm for different perturbations $e$ and tolerances tol

Inputs Results

\begin{tabular}{|c|c|c|c|c|}
\hline tol & $e$ & \multicolumn{2}{|c|}{ Branch taken (right/wrong) } & Inverse computed \\
\hline $10^{\wedge}-20$ & $10^{\wedge}-1$ & 2 & (right) & Correct \\
\hline $10^{\wedge}-20$ & $10^{\wedge}-8$ & 2 & (right) & Correct \\
\hline $10^{\wedge}-20$ & $10^{\wedge}-10$ & 2 & (right) & Correct \\
\hline $10^{\wedge}-20$ & $10^{\wedge}-12$ & 1 & (wrong) & Incorrect \\
\hline $10^{\wedge}-12$ & $10^{\wedge}-5$ & 2 & (right) & Correct \\
\hline $10^{\wedge}-10$ & $10^{\wedge} 0$ & 2 & (right) & Correct \\
\hline $10^{\wedge}-10$ & $10^{\wedge}-5$ & 2 & (right) & Correct \\
\hline $10^{\wedge}-10$ & $10^{\wedge}-6$ & 1 & (wrong) & Incorrect \\
\hline $10^{\wedge}-8$ & $10^{\wedge}-4$ & 2 & (right) & Correct \\
\hline $10^{\wedge}-8$ & $10^{\wedge}-5$ & 1 & (wrong) & Incorrect \\
\hline $10^{\wedge}-7$ & $10^{\wedge}-3$ & 2 & (right) & Correct \\
\hline $10^{\wedge}-6$ & $10^{\wedge}-3$ & 2 & (right) & Correct \\
\hline $10^{\wedge}-5$ & $10^{\wedge}-3$ & 1 & (wrong) & Incorrect \\
\hline
\end{tabular}

